\title{
Universal correlations and coherence in quasi-two-dimensional trapped Bose gases
}

\author{
Markus Holzmann ${ }^{1}$, Maguelonne Chevallier ${ }^{2}$, Werner Krauth ${ }^{2}$ \\ ${ }^{1}$ LPTMC, Université Pierre et Marie Curie, 4 Place Jussieu, 75005 Paris, \\ France; and LPMMC, CNRS-UJF, BP 166, 38042 Grenoble, France* and \\ ${ }^{2}$ CNRS-Laboratoire de Physique Statistique, Ecole Normale Supérieure, \\ 24 rue Lhomond, 75231 Paris Cedex 05, France
}

(Dated: October 11, 2018)

\begin{abstract}
We study the quasi-two-dimensional Bose gas in harmonic traps at temperatures above the Kosterlitz-Thouless transition, where the gas is in the normal phase. We show that mean-field theory takes into account the dominant interaction effects for experimentally relevant trap geometries. Comparing with Quantum Monte Carlo calculations, we quantify the onset of the fluctuation regime, where correlations beyond mean-field become important. Although the density profile depends on the microscopic parameters of the system, we show that the correlation density (the difference between the exact and the mean-field density) is accurately described by a universal expression, obtained from classical-field calculations of the homogeneous strictly two-dimensional gas. Deviations from universality, due to the finite value of the interaction or to the trap geometry, are shown to be small for current experiments. We further study coherence and pair correlations on a microscopic scale. Finite-size effects in the off-diagonal density matrix allows us to characterize the cross-over from Kosterlitz-Thouless to Bose-Einstein behavior for small particle numbers. BoseEinstein condensation occurs below a characteristic number of particles which rapidly diverges with vanishing interactions.
\end{abstract}

PACS numbers: 03.75.Hh,05.30.Jp

\section{INTRODUCTION}

In recent years, several experiments [1,2] studied twodimensional ultra-cold atomic gases from the normal phase down in temperature to the Kosterlitz-Thouless transition [3] and into the low-temperature superfluid phase. The interference of two simultaneously prepared two-dimensional gases evidenced the presence of vortices [1]. Related experiments investigated interaction and correlation effects $[2,4]$ in the density profile and in coherence patterns. For a quantitative description of the Kosterlitz-Thouless transition and of the interaction effects, it proved necessary to account for the quasi-twodimensional nature of the gas, that is to include thermal excitations in the strongly confined $z$-axis in addition to the weak trapping potential in the $x y$-plane $[5,6]$.

In weakly interacting two-dimensional Bose gases, the Kosterlitz-Thouless phase transition occurs at relatively high phase-space density (number of atoms per phase-space cell $\left.\lambda_{T}^{2}=2 \pi \hbar^{2} / m T\right)$. This density is $n_{c} \lambda_{T}^{2} \simeq \log \left(\xi_{n} / \tilde{g}\right)[7-9]$, where $\tilde{g}$ characterizes the twodimensional interaction strength, $T$ is the temperature, $m$ the mass of the atoms, and $n$ the density. The coefficient $\xi_{n}=380 \pm 3$ was determined numerically using classical-field simulations [9]. For the ENS experiment of Hadzibabic et al. [1], the critical phase-space density is $n_{c} \lambda_{\mathrm{T}}^{2} \sim 8$ in the center of the trap, whereas in the NIST experiment of Cladé et al. [2], $n_{c} \lambda_{\mathrm{T}}^{2}$ is close to 10. For phase-space densities between one and the

\footnotetext{
*Electronic address: markus@lptmc.jussieu.fr
}

$\dagger$ Electronic address: werner.krauth@ens.fr critical number, the gas is quantum degenerate yet normal. The atoms within one phase-space cell are indistinguishable. They lose their particle properties and acquire the characteristics of a field. The mean-field description of particles interacting with a local atomic density $n(r)$ may further be modified through correlations and fluctuations. Quantum correlations can be several times larger than the scale $\lambda_{\mathrm{T}}$. This gives rise to "quasi-condensate" behavior inside the normal phase.

In this paper, we study the quantum-degenerate regime at high phase-space density in the normal phase. We first discuss the peculiar quasi-two-dimensional thermodynamic limit where, as the number of particles in the gas is increased, the interactions and the lattice geometry are scaled such that a finite fraction of all particles are in the excited states of the system. In this thermodynamic limit, the Kosterlitz-Thouless transition takes place at a temperature comparable to the Bose-Einstein transition temperature in the non-interacting case, and the local-density approximation becomes exact. We first clarify the relation between different recent versions of quasi-two-dimensional mean-field theory $[6,10,11]$ in the local-density approximation (LDA), and also determine the finite-size corrections to the LDA. We compare meanfield theory to a numerically exact solution obtained by path-integral Quantum Monte Carlo (QMC) calculations with up to $N \gtrsim 10^{5}$ interacting particles in a harmonic trap with parameters chosen to fit the experiments. We concentrate on the correlation density, the difference between the exact density and the mean-field density at equal chemical potential, and show that it is essentially a universal function, independent of microscopic details. Within classical-field theory, the correlation density is obtained from a reparametrization of known results for 
the strictly two-dimensional homogeneous system [12]. The classical-field results hold for small interaction parameters $\tilde{g} \rightarrow 0$, but our full QMC solution accounts for corrections. We compute the correlation density by QMC and show that it is largely independent of the trap geometry, the temperature, and the interaction strength.

We also study off-diagonal coherence properties, and the density-density correlation function of the quasi-twodimensional gas. It is well known that even at high temperature, bosonic bunching effects enhance the paircorrelation function on length scales below $\lambda_{\mathrm{T}}$ which for the ideal Bose gas approaches the characteristic value $2 n^{2}$ at vanishing separation. In our case, interference in the $z$-direction reduces the in-plane density fluctuations even for an ideal gas and within mean-field theory, and the reduction of the pair correlations from $2 n^{2}$ no longer proves the presence of beyond-mean-field effects.

We finally discuss finite-size effects in the quasi-twodimensional Bose gas. For the density profile, they are not very large, but we point out their great role for off-diagonal correlations. The latter are responsible for a cross-over between the physics of Bose-Einstein condensation at small particle number and the KosterlitzThouless physics for larger systems; both regimes are of relevance for current experiments. This cross-over takes place at a particle number $N \sim \tilde{g}^{-2}$ which grows very rapidly as the interaction in the gas diminishes.

\section{SYSTEM PARAMETERS AND MEAN-FIELD DESCRIPTION}

\section{A. Quasi-two-dimensional thermodynamics}

We consider $N$ bosons in a three-dimensional pancakeshaped harmonic potential with parameters $\omega_{x}=\omega_{y}=\omega$ and $\omega_{z} \gg \omega$ at inverse temperature $\beta=1 / T$. The $z$ variable is separate from $x$ and $y$, and we denote the three-dimensional vectors as $\vec{r}=(\mathbf{r}, z)$, and write twodimensional vectors as $\mathbf{r}=(x, y)$, and $r=|\mathbf{r}|$.

The quasi-two-dimensional regime of the Bose gas [6] is defined through a particular thermodynamic limit $N \rightarrow$ $\infty$, where the temperature is a fixed fraction $t \equiv T / T_{\mathrm{BEC}}^{2 \mathrm{~d}}$ of the Bose-Einstein transition temperature of the ideal two-dimensional Bose gas, $T_{\mathrm{BEC}}^{2 \mathrm{~d}}=\sqrt{6 N} \hbar \omega / \pi$. Although the two-dimensional trapped Bose gas undergoes a BoseEinstein transition only for zero interactions, $T_{\mathrm{BEC}}^{2 \mathrm{~d}}$ still sets the scale for the Kosterlitz-Thouless transition in the interacting gas $[6,8]$. In the quasi-two-dimensional regime, a finite fraction of atoms remains in excited states in $z$. The excitation energy is scaled as $\hbar \omega_{z} \propto T_{\mathrm{BEC}}^{2 \mathrm{~d}}$, which implies that $\omega_{z}$ increases as $N^{1 / 2}$ in the thermodynamic limit.

Interatomic collisions are intrinsically threedimensional. Here, we consider the experimentally relevant case where the range of the scattering potential $r_{0}$ is much smaller than the typical lateral extension $l_{z}=\left(m \omega_{z} / \hbar\right)^{-1 / 2}$, and also where $r_{0}$ is much smaller than the inter-particle distances. The interactions are then described by the three-dimensional $s$-wave scattering length $a_{s}$, and one may characterize the quasi-two-dimensional gas through a bare effective two-dimensional interaction strength, $\tilde{g}$,

$$
\begin{aligned}
& \tilde{g}=\frac{m g}{\hbar^{2}} \int \mathrm{d} z\left[\psi_{0}(z)\right]^{4} \\
& g=\frac{4 \pi \hbar^{2} a_{s}}{m},
\end{aligned}
$$

where $\psi_{0}(z)$ is the unperturbed ground state of the confining potential and $g$ is the usual three-dimensional coupling constant. For a harmonic confinement, $\tilde{g}=$ $\sqrt{8 \pi} a_{s} / l_{z}$, and $a_{s} / l_{z}$ must be kept constant in the thermodynamic limit to obtain a fixed two-dimensional interaction strength.

Quasi-two-dimensional scattering amplitudes depend logarithmically on energy, $\epsilon$, in terms of a universal function of $a_{s} / l_{z}$ and of $\epsilon / \hbar \omega_{z} \sim T / \omega_{z}$, which are both kept constant in the quasi-two-dimensional thermodynamic limit. The logarithmic energy dependence yields small corrections of order $\left(a_{s} / l_{z}\right)^{2}[13-15]$ to the bare interaction $\tilde{g}$. They can be neglected in the following.

The scaling behavior in the quasi-two-dimensional limit corresponds to the following reduced variables:

$$
\begin{array}{ll}
\tilde{r}=r / l_{T} & \tilde{z}=z / l_{z} \\
t=T / T_{\mathrm{BEC}}^{2 \mathrm{~d}} & \tilde{\omega}_{z}=\hbar \omega_{z} / T_{\mathrm{BEC}}^{2 \mathrm{~d}} \\
\tilde{n}=n \lambda_{\mathrm{T}}^{2} & \tilde{g}=m g /\left(\sqrt{2 \pi} l_{z} \hbar^{2}\right),
\end{array}
$$

where $l_{T}=\left(T / m \omega^{2}\right)^{1 / 2}$ is the thermal extension in the plane. The quasi-two-dimensional limit consists in taking $N \rightarrow \infty$, with $t, \tilde{\omega}_{z}$ and $\tilde{g}$ all constant. In this limit, $l_{T} / \lambda_{\mathrm{T}}=t \sqrt{3 N / \pi^{3}} \gg 1$, so that macroscopic and microscopic length scales separate, and the scaling of the three-dimensional density, $n_{3 d}$, is at constant

$$
\tilde{n}_{3 d}=n_{3 d} \lambda_{\mathrm{T}}^{2} l_{z} .
$$

In reduced variables, the normalization condition $N=$ $\int \mathrm{d} \mathbf{r} n(\mathbf{r})=\left(l_{T} / \lambda_{\mathrm{T}}\right)^{2} \int \mathrm{d} \tilde{\mathbf{r}} \tilde{n}(\tilde{\mathbf{r}})$ is expressed as

$$
\int_{0}^{\infty} \mathrm{d} \tilde{r} \tilde{r} \tilde{n}(\tilde{r})=\frac{\pi^{2}}{6 t^{2}}
$$

The local-density approximation becomes exact in the quasi-two-dimensional limit.

In the ENS experiment, ${ }^{87} \mathrm{Rb}$ atoms are trapped at temperatures $T \approx 50-100 \mathrm{nK}$. The in-plane trapping frequencies are $\omega /(2 \pi) \approx 50 \mathrm{~Hz}$ whereas the confinement is of order $\omega_{z} /(2 \pi) \approx 3 \mathrm{kHz}$. With $N \sim 2 \cdot 10^{4}$ atoms trapped inside one plane, typical parameters are $T_{\mathrm{BEC}}^{2 \mathrm{~d}} \approx 300 \mathrm{nK}$ (using $\hbar / k_{B} \simeq 7.64 \cdot 10^{-3} \mathrm{nKs}$ ), so that $\tilde{\omega}_{z} \approx 0.44-0.55$. The scattering length $a_{s}=5.2 \mathrm{~nm}$ leads to an effective coupling constant $\tilde{g}=0.13$, using $\hbar / m \simeq 6.3 \cdot 10^{-8} \mathrm{~m}^{2} \mathrm{~s}^{-1} A^{-1}$, where $A$ is the atomic mass number. In the NIST experiment, sodium atoms at $T \approx$ $T_{\mathrm{BEC}}^{2 \mathrm{~d}} \approx 100 \mathrm{nK}$ are confined by harmonic trapping potentials with $\omega /(2 \pi) \approx 20 \mathrm{~Hz}$, and $\omega_{z} /(2 \pi) \approx 1 \mathrm{kHz}$. This is 
described by reduced parameters $\tilde{g}=0.02$ and $\tilde{\omega}_{z}=0.50$. The critical densities are $\tilde{n}_{c} \approx \log (380 / \tilde{g}) \approx 8.2$ for the ENS parameters, somewhat lower than the NIST value $\tilde{n}_{c} \approx 9.9$. Using the quasi-two-dimensional mean-field estimates of Ref. [6], the Kosterlitz-Thouless temperatures are located at $t_{\mathrm{KT}} \equiv T_{\mathrm{KT}} / T_{\mathrm{BEC}}^{2 \mathrm{~d}} \approx 0.69$ and $t_{\mathrm{KT}} \approx 0.74$, respectively.

The quasi-two-dimensional limit describes a kinematically two-dimensional gas, whose extension in the $z$ direction is of the order of the thermal wavelength $\lambda_{\mathrm{T}}$. As $\tilde{\omega}_{z} \equiv \lambda_{\mathrm{T}}^{2} /\left(2 \pi t l_{z}^{2}\right)$ is decreased, a system at finite $N$ turns three-dimensional. This is already the case for the ideal quasi-two-dimensional gas (with $\tilde{g}=0$ ) where the BoseEinstein transition temperature crosses over from twodimensional to three-dimensional behavior as a function of $\tilde{\omega}_{z}$, with asymptotic behavior given by

$$
t_{\mathrm{BEC}} \sim \begin{cases}{\left[\frac{\zeta(2)}{\zeta(3)}\right]^{1 / 3} \tilde{\omega}_{z}^{1 / 3}-\frac{1}{6} \frac{\zeta(2)}{\zeta(3)} \tilde{\omega}_{z}} & \text { for } \tilde{\omega}_{z} \ll 1 \\ 1-\frac{1}{2 \zeta(2)^{3 / 2}} \exp \left(-\tilde{\omega}_{z}\right) & \text { for } \tilde{\omega}_{z} \gg 1\end{cases}
$$

(see [6]). In Eq. (6), the first term for $\tilde{\omega}_{z} \ll 1$ describes three-dimensional Bose-Einstein condensation in an anisotropic trapping potential.

For the interacting Bose gas, the nature of the Kosterlitz-Thouless transition in two dimensions differs from the Bose-Einstein transition of the threedimensional gas. For large $\tilde{\omega}_{z}$, universal features of the Kosterlitz-Thouless transition are preserved, but the density profiles and the value of the Kosterlitz-Thouless transition temperature depend on $\tilde{\omega}_{z}$ and $\tilde{g}[5,6]$. For small confinement strength $\tilde{\omega}_{z}$, a dimensional cross-over between the two-dimensional Kosterlitz-Thouless transition and the three-dimensional Bose-Einstein condensation takes place at particle numbers such that the level spacing in the confined direction is comparable to the (two-dimensional) correlation energies, $t \tilde{\omega}_{z} \lesssim \tilde{g} \tilde{n} / \pi$.

The quasi-two-dimensional limit differs from the "experimentalist's" thermodynamic limit where the atom number is increased in a fixed trap geometry, and at constant temperature. In this situation, the ratio between the microscopic and the macroscopic length scales, $l_{T} / \lambda_{T}=T /(\hbar \omega \sqrt{2 \pi})$, remains constant and finite. The number of particles in any region of nearly constant density remains also finite so that, in contrast to the quasitwo-dimensional thermodynamic limit, corrections to the LDA persist.

\section{B. N-body and mean-field Hamiltonians}

The gas specified in Section II A is described by the Hamiltonian

$$
\begin{aligned}
H & =H_{0}+V \\
H_{0} & =\sum_{i=1}^{N}\left[-\frac{\hbar^{2} \nabla_{i}^{2}}{2 m}+\frac{1}{2} m\left[\omega^{2} \mathbf{r}_{i}^{2}+\omega_{z}^{2} z_{i}^{2}\right]\right], \\
V & =\sum_{i<j=1}^{N} v\left(\left|\vec{r}_{i}-\vec{r}_{j}\right|\right),
\end{aligned}
$$

where $v$ is the three-dimensional interaction potential. We compute the $N$-body density matrix at finite temperature using three-dimensional path-integral QMC methods. We thus obtain all the thermodynamic observables $[5,16,17]$ for up to $N=10^{6}$. QMC calculations have clearly demonstrated the presence of a KosterlitzThouless transition [6] for parameters corresponding to the ENS experiment.

In the mean-field approximation, one replaces the $N$ body interaction between atoms in Eq. (9) by an effective single-particle potential. The mean-field Hamiltonian writes

$$
H_{\mathrm{mf}}=H_{0}+V_{\mathrm{mf}}
$$

where

$$
V_{\mathrm{mf}}=\sum_{i=1}^{N} 2 g n_{3 d}\left(\vec{r}_{i}\right)-g \int \mathrm{d} \vec{r}\left[n_{3 d}(\vec{r})\right]^{2}
$$

is the mean-field potential energy. From the corresponding partition function in the canonical or grandcanonical ensemble, all thermodynamic quantities can be calculated. The three-dimensional density $n_{3 d}(\vec{r})$ inside the mean-field potential must be determined selfconsistently. In all situations treated in the present paper, self-consistency is reached through straightforward iteration.

Mean-field theory leads to an effective Schrödinger equation for the single-particle wavefunction, $\Psi_{j}(\vec{r})$, of energy $\epsilon_{j}$

$$
\begin{array}{r}
{\left[-\frac{\hbar^{2} \nabla_{\mathbf{r}}^{2}}{2 m}+\frac{1}{2} m \omega^{2} r^{2}-\frac{\hbar^{2} \partial^{2}}{2 m \partial z^{2}}+\frac{1}{2} m \omega_{z}^{2} z^{2}+2 g n_{3 d}(\vec{r})\right]} \\
\Psi_{j}(\vec{r})=\epsilon_{j} \Psi_{j}(\vec{r})
\end{array}
$$

together with the total density

$$
n_{3 d}(\vec{r})=\sum_{i} \frac{\Psi_{j}^{*}(\vec{r}) \Psi_{j}(\vec{r})}{e^{\beta\left(\mu-\epsilon_{j}\right)}-1} .
$$

The exact solution of the mean-field eigenfunctions and eigenvalues for finite systems, is rather involved, but considerably simplifies in the local-density approximation.

At finite $N$, in the canonical ensemble, we solve the mean-field equations through a Quantum Monte Carlo 
simulation with $N$ particles which avoids an explicit calculation of all eigenfunctions. In contrast to the usual interaction energy within QMC, consisting, in general, of a pair interaction potential, the mean-field interaction energy is simply given in terms of an anisotropic, singleparticle potential proportional to the three-dimensional density profile, $n_{3 d}(\vec{r})$, as in Eqs (10) and (11). This interaction potential must be obtained self-consistently as usual in mean-field. Once self-consistency in the density is reached, one can compute correlation functions and off-diagonal elements of the reduced one-body density matrix.

\section{Mean-field: Local-density approximation}

Mean-field theory simplifies in the quasi-twodimensional thermodynamic limit, as the local-density approximation then becomes exact. This is because the natural length scale of the system, $\lambda_{\mathrm{T}}$, separates from the macroscopic scale $l_{T}$ of variation of the density $\left(\lambda_{\mathrm{T}} / l_{T} \rightarrow 0\right)$. The particle numbers inside a region of constant density diverges. The decoupling of length scales implies that the $x y$-dependence of the single particle wavefunctions in Eq. (12) separates in the thermodynamic limit. Using scaled variables, Eq. (3), this yields

$$
\begin{aligned}
{\left[-\frac{1}{2} \frac{d^{2}}{d \tilde{z}^{2}}+\frac{1}{2} \tilde{z}^{2}+\frac{2 \tilde{g} t}{\sqrt{2 \pi} \tilde{\omega}_{z}} \tilde{n}_{3 d}^{\operatorname{mf}}(\tilde{r}, \tilde{z})\right] } \\
\tilde{\phi}_{\nu}(\tilde{r}, \tilde{z})=\frac{\tilde{\epsilon}_{\nu}(\tilde{r})}{\tilde{\omega}_{z}} \tilde{\phi}_{\nu}(\tilde{r}, \tilde{z})
\end{aligned}
$$

for the eigenfunctions, $\tilde{\phi}_{\nu}(\tilde{r}, \tilde{z})$, and eigenvalues, $\tilde{\epsilon}_{\nu}(\tilde{r})$, in the confined direction, at a given radial distance, $\tilde{r}$. The reduced local density $\tilde{n}_{3 d}^{\mathrm{mf}}$ is given by the normalized wavefunctions $\tilde{\phi}(\tilde{r}, \tilde{z})$ :

$$
\begin{aligned}
\tilde{n}_{3 d}^{\mathrm{mf}}(\tilde{r}, \tilde{z}) & =\sum_{\nu} \tilde{\phi}_{\nu}^{2}(\tilde{r}, \tilde{z}) \tilde{n}_{\nu}^{\mathrm{mf}}(\tilde{r}) \\
\tilde{n}_{\nu}^{\mathrm{mf}}(\tilde{r}) & =-\log \left[1-\exp \left(\tilde{\mu}(\tilde{r})-\tilde{\epsilon}_{\nu}(\tilde{r}) / t\right)\right] .
\end{aligned}
$$

The position-dependence in Eq. (14) and Eq. (15) only enters parametrically through the $\tilde{r}$-dependence of the chemical potential,

$$
\tilde{\mu}(\tilde{r})=\tilde{\mu}-\frac{\tilde{r}^{2}}{2}
$$

and the local-density approximation becomes exact in the quasi-two-dimensional thermodynamic limit. Within LDA, density profiles (as in Fig. 1) are directly related to the equation of state $\tilde{n}(\tilde{\mu})$ of a quasi-two-dimensional system, which is homogeneous in the $x y$-plane.

The Schrödinger equation of Eq. (14) is conveniently written in the basis $\left\{\psi_{0}, \psi_{1}, \ldots, \psi_{n}, \ldots\right\}$ of the onedimensional harmonic oscillator with $\omega=m=1$, as it diagonalizes Eq. (14) for $\tilde{g}=0$. Using

$$
\tilde{\phi}_{\nu}(\tilde{z})=\sum_{\mu} a_{\mu \nu} \psi_{\mu}(\tilde{z})
$$

(where we have dropped the index corresponding to $\tilde{r}$ or, equivalently, to $\tilde{\mu}$ ), we can write it as a matrix equation

$$
\left(\mathbf{A}-\frac{\tilde{\epsilon}_{\nu}}{\tilde{\omega}_{z}}\right) \mathbf{a}_{\nu}=0
$$

with eigenvalues $\tilde{\epsilon}_{\nu} / \tilde{\omega}_{z}$ and eigenvectors $\mathbf{a}_{\nu}=$ $\left\{a_{0 \nu}, \ldots, a_{n \nu}\right\}$, of the $(n+1) \times(n+1)$ matrix

$$
\mathbf{A}_{\mu \nu}=\nu \delta_{\mu \nu}+\frac{2 \tilde{g} t}{\sqrt{2 \pi} \tilde{\omega}_{z}} \int \mathrm{d} \tilde{z} \psi_{\mu}(\tilde{z}) \tilde{n}_{3 d}^{\operatorname{mf}}(\tilde{\mu}, \tilde{z}) \psi_{\nu}(\tilde{z})
$$

(where $0 \leq \mu, \nu \leq n$ ) and the density $\tilde{n}_{3 d}^{\operatorname{mf}}(\tilde{\mu}, \tilde{z})=$ $-\sum_{\nu} \tilde{\phi}_{\nu}^{2}(\tilde{z}) \log \left[1-\exp \left(\tilde{\mu}-\tilde{\epsilon}_{\nu} / t\right)\right]$. The wavefunctions $\psi_{\nu}$ are easily programmed (see, e.g., [18] sect. 3.1), and the self-consistent mean-field solutions at each value of $\tilde{\mu}$ can be found via iterated matrix diagonalization.

This full solution of the LDA mean-field equations is analogous to the one in Ref.[10]. The mean-field version used in [11], however, neglects the off-diagonal couplings in $A_{\nu \mu}$ with $\nu \neq \mu$. In [6], we used a simplified mean-field potential in order to reach explicit analytical expressions. These different mean-field approximations essentially coincide at all relevant temperatures [11], but ground-state occupations in $z$ slightly differ. Further replacing the coupling constant $\tilde{g}$ by $\tilde{g} \tanh ^{1 / 2}\left(\tilde{\omega}_{z} / 2 t\right)$ has allowed us, in Ref. [6], to improve the agreement with the QMC results close to the transition. This is because mean-field theory overestimates the effect of the interactions in the fluctuation regime. In the following we always quantify beyond-mean-field corrections with respect to the full LDA solution of Eq. (19).

\section{CORRELATION DENSITY AND UNIVERSALITY}

Comparisons between the QMC and the mean-field density profiles are shown in Fig. 1 for the ENS parameters at reduced temperature $t=0.71$, slightly above the Kosterlitz-Thouless temperature. Finite-size effects as well as deviations from mean-field theory are visible for $n \lambda_{\mathrm{T}}^{2} \gtrsim 5$. In this section, we concentrate on correlation corrections to mean-field theory in the thermodynamic limit and postpone the discussion of finite-size effects to Section IV. We analyze the QMC density profiles within the validity of the LDA, Eq. (16), and compare QMC and mean-field densities at the same local chemical potential [29] which defines the correlation density, $\Delta \tilde{n}$,

$$
\Delta \tilde{n}(\tilde{\mu})=\tilde{n}(\tilde{\mu})-\tilde{n}_{\mathrm{mf}}(\tilde{\mu}) .
$$

As in experiments, the chemical potential is not a control parameter of the QMC calculation, but it can be obtained 
from a fit of the wings of the density profile with $\tilde{n} \lesssim 1$ to the mean-field equation of state.

Mean-field effects take into account the dominant interaction effects which, in particular, determine shape and energies of the ground and excited states in the tightly confined direction. One expects that correlation effects do not modify these high-energy modes, but merely affects the low-energy distribution of $x y$-modes inside the confining ground state, $\tilde{\phi}_{0}(\tilde{z})$. This assumption is supported by a direct comparison of the normalized density distribution in $\tilde{z}$ between the QMC solution and the mean-field approximation (inset of Fig. 1) at different radial distances, $\tilde{r}$. For small $\tilde{r}$, the ground state of the confining potential is strongly populated. For larger $\tilde{r}$, higher modes of the one-dimensional harmonic oscillator are thermally occupied, and the density distribution broadens. However, the normalized density profile in $\tilde{z}$ is everywhere well described by mean-field theory, and correlation effects hardly modify the mode structure in the confined direction.

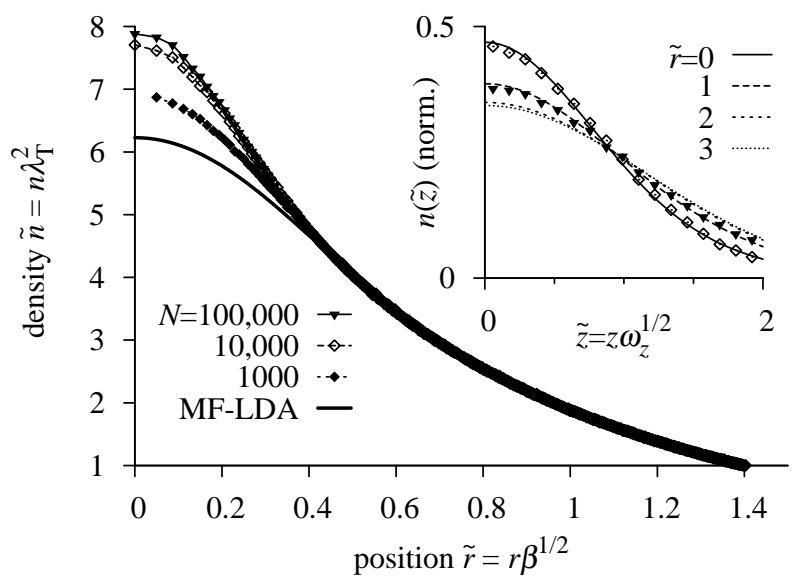

FIG. 1: QMC density profile for the ENS parameters $(\tilde{g}=$ $\left.0.13, \tilde{\omega}_{z}=0.55\right)$ at temperature $t=T / T_{\mathrm{BEC}}^{2 \mathrm{~d}}=0.71$ for different values of the particle number, compared to the LDA mean-field solution of Section IIB at the same total number of particles. The inset shows the density distribution $n(\tilde{z})$ at different values of the radial distance $\tilde{r}$.

In the homogeneous two-dimensional gas, corrections to mean-field theory at small $\tilde{g}$ are described by classicalfield theory, and correlation effects in the density profile can be expressed in terms of a universal function of $\beta\left(\mu-2 g n_{\mathrm{mf}}\right)[8,12]$. In a quasi-two-dimensional geometry, the corresponding relevant quantity is given by the local mean-field gap $\Delta_{\mathrm{mf}}$ between the local ground-state energy in the confining potential and the local chemical potential,

$$
\Delta_{\mathrm{mf}}(\tilde{r})=\tilde{\epsilon}_{0}(\tilde{r}) / t-\tilde{\mu}(\tilde{r}) .
$$

Within mean-field theory, it fixes the local $x y$-density in the ground state of the confining potential

$$
\tilde{n}_{0}^{\mathrm{mf}}(\tilde{r})=-\log \left(1-\mathrm{e}^{-\Delta_{\mathrm{mf}}(\tilde{r})}\right) .
$$

In the strictly two-dimensional limit, we have

$$
\Delta_{\mathrm{mf}} \rightarrow \beta\left(2 \tilde{g} \hbar^{2} n_{\mathrm{mf}} / m-\mu\right)=\tilde{g} \tilde{n}_{\mathrm{mf}} / \pi-\tilde{\mu}
$$

where $n_{\mathrm{mf}}$ is the total mean-field density. Deviations are noticeable for large densities, as illustrated in Fig. 2 (we have absorbed the zero-point energy $\tilde{\omega}_{z} /(2 t)$ in the chemical potential). Within LDA, we expect that the correlation density $\Delta \tilde{n}$ coincides to leading order in $\tilde{g}$ with the classical-field-theory results of the homogeneous strictly two-dimensional system [12], expressed as functions of the mean-field gap $\Delta_{\mathrm{mf}}[30]$.

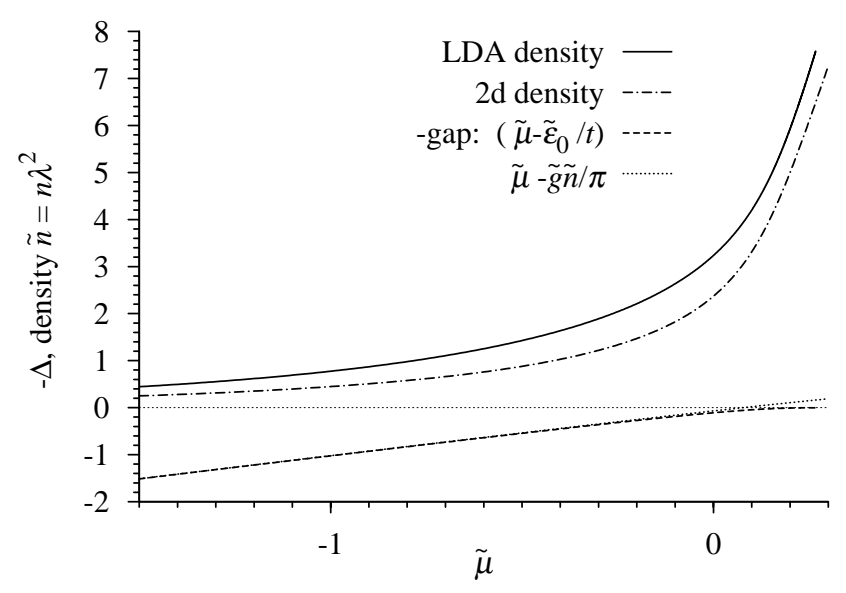

FIG. 2: Mean-field equation of state and gap $\Delta_{\mathrm{mf}}$ for ENS parameters $\tilde{g}=0.13, \tilde{\omega}_{z}=0.55, t=0.71$. The gap differs from the approximation $\tilde{\mu}-\tilde{g} \tilde{n} / \pi$ only at high density. The 2d mean-field curve (see Eqs (22) and (23)) illustrates the dependence of the equation of state on microscopic parameters.

In Ref. [12], the critical density $\tilde{n}_{c}$ and chemical potential $\tilde{\mu}_{c}$ at the Kosterlitz-Thouless transition were determined to

$$
\begin{aligned}
& \tilde{n}_{c}=\log \frac{\xi_{n}}{\tilde{g}}, \quad \xi_{n}=380 \pm 3 \\
& \tilde{\mu}_{c}=\frac{\tilde{g}}{\pi} \log \frac{\xi_{\mu}}{\tilde{g}}, \quad \xi_{\mu}=13.2 \pm 0.4
\end{aligned}
$$

and the equation of state in the neighborhood of the transition was written as

$$
\tilde{n}-\tilde{n}_{c}=2 \pi \lambda(X), \text { with } X=\left(\tilde{\mu}-\tilde{\mu}_{c}\right) / \tilde{g} .
$$

The function $\lambda(X)$ was tabulated. Consistent with the classical-field approximation, we can expand Eq. (22) to leading order in $\Delta_{\mathrm{mf}}$,

$$
\tilde{n}_{0}^{\mathrm{mf}}\left(\Delta_{\mathrm{mf}}\right)=-\log \left(\Delta_{\mathrm{mf}}\right),
$$

so that we can express $X$ through $\Delta_{\mathrm{mf}}$ :

$$
\begin{aligned}
X\left(\Delta_{\mathrm{mf}}\right)=\frac{\tilde{n}_{\mathrm{mf}}}{\pi} & -\frac{\Delta_{\mathrm{mf}}}{\tilde{g}}-\frac{\tilde{\mu}_{c}}{\tilde{g}} \\
& =-\left[\frac{\Delta_{\mathrm{mf}}}{\tilde{g}}+\frac{1}{\pi} \log \left(\xi_{\mu} \frac{\Delta_{\mathrm{mf}}}{\tilde{g}}\right)\right] .
\end{aligned}
$$


Thus, we obtain the correlation density as a function of $\Delta_{\mathrm{mf}}:$

$$
\Delta \tilde{n}=2 \pi \lambda\left[X\left(\Delta_{\mathrm{mf}}\right)\right]+\log \left(\xi_{n} \Delta_{\mathrm{mf}} / \tilde{g}\right),
$$

and a straightforward inversion of Eq. (28) allows us to translate the data of [12] in order to obtain the correlation density as a function of the mean-field gap. An empirical interpolation of the numerical data with $\lesssim 10 \%$ error inside the fluctuation regime, $\Delta_{\mathrm{mf}}^{c} \leq \Delta_{\mathrm{mf}} \leq \Delta_{\mathrm{mf}}^{f}$ $\left(\Delta_{\mathrm{mf}}^{c}\right.$ and $\Delta_{\mathrm{mf}}^{f}$ are defined below $)$, is given by

$$
\Delta \tilde{n}\left(\Delta_{\mathrm{mf}} / \tilde{g}\right) \simeq \frac{1}{5}\left(-1+\frac{1}{\Delta_{\mathrm{mf}} / \tilde{g}}\right) \frac{1}{1+\pi \Delta_{\mathrm{mf}}^{2} / \tilde{g}^{2}}
$$

where positivity is imposed since, within classical field theory, the correlation density must be positive and of order $\left(\Delta_{\mathrm{mf}} / \tilde{g}\right)^{-2}$ for $\Delta_{\mathrm{mf}} / \tilde{g} \rightarrow \infty$.

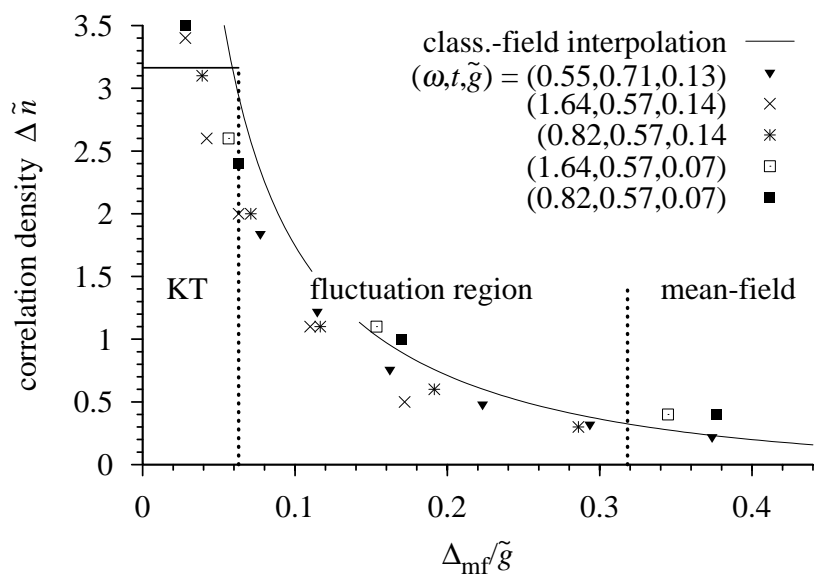

FIG. 3: Correlation density $\Delta \tilde{n}$ vs. rescaled mean-field gap $\Delta_{\mathrm{mf}} / \tilde{g}$. QMC data for various interaction strengths and confinements $\tilde{\omega}_{z}$ are compared with the interpolation Eq. (30) of classical-field results [12].

In Fig. 3, we plot the classical-field results for the correlation density as a function of the rescaled meanfield gap. We also indicate the onset of the KosterlitzThouless transition $\left(\tilde{\mu}=\tilde{\mu}_{c}, X=0\right.$ in Eq. (29)) at $\Delta_{\mathrm{mf}}^{c} / \tilde{g}=0.0623$, which yields $\Delta \tilde{n}_{c}=3.164$. The correlation density in the normal phase is thus finite for all interactions, whereas the mean-field density diverges as $\tilde{n}_{\mathrm{mf}}=-\log \Delta_{\mathrm{mf}}^{c} \propto-\log g$ for small interactions at $T_{\mathrm{KT}}$. In Fig. 3, we furthermore compare the classical-field data for the correlation density with the results of QMC simulations of quasi-two-dimensional trapped Bose gases with different coupling constants $\tilde{g}$ and confinement strengths $\tilde{\omega}_{z}$. The QMC data illustrates that the external trapping and the quasi-two-dimensional geometry preserve universality in the experimental parameter regime. However, the finite coupling constant $\tilde{g}$ introduces small deviations due to quantum corrections.

From Fig. 3, we further see that the correlation density is reduced to roughly $10 \%$ of its critical value for mean-field gaps $\Delta_{\mathrm{mf}}^{f} \simeq \tilde{g} / \pi$. Thus, only densities with $\tilde{n} \gtrsim \tilde{n}_{f} \approx \tilde{n}_{\mathrm{mf}}\left(\Delta_{\mathrm{mf}} \approx \tilde{g} / \pi\right)$ are significantly affected by correlations, and $\tilde{n}_{f}$ can be considered as the boundary of the fluctuation regime. In fact, perturbation theory fails inside this regime. For a strictly two-dimensional system, we have

$$
\tilde{n}_{f} \approx \log (\pi / \tilde{g}),
$$

and the fluctuation regime is reached for densities $\tilde{n} \gtrsim$ $\tilde{n}_{f}$. Outside the fluctuation regime, $\tilde{n} \lesssim \tilde{n}_{f}$, mean-field theory is rather accurate, and can be improved perturbatively, if necessary.

To understand this criterion, which is important for the Kosterlitz-Thouless to Bose-Einstein cross-over at small $N$ (see Section IV), we briefly analyze the perturbative structure of the two-dimensional single-particle Green's function beyond mean-field theory [8]. Within classical-field theory, second-order diagrams are ultraviolet convergent. Each additional higher order brings in a factor $\hbar^{2} \tilde{g} / m$ for the interaction vertex, one integration over two-momenta, a factor $T$, and two Green's functions (the internal lines). Dimensional analysis of the integrals involved shows that each vertex insertion adds a factor $\tilde{g} / \Delta_{\mathrm{mf}}$. This implies that perturbation theory fails for $\tilde{g} / \Delta_{\mathrm{mf}} \gtrsim 1$.

For lower densities, $1 \lesssim \tilde{n} \lesssim \tilde{n}_{f}$, the gas is quantum degenerate, yet it is accurately described by mean-field theory. In contrast to fully three-dimensional gases, the quantum-degenerate regime can be rather broad in two dimensions for gases with $\tilde{g} \ll 1$. In this regime, the density, yet normal, is no longer given by a thermal Gaussian distribution. This was observed in the NIST experiment [2] where $\tilde{n}_{f} \simeq 5$. In Fig. 4 , we illustrate this effect via the approximations for the tails of the distribution $\tilde{n}(\tilde{r})$ with between one and five Gaussians, as

$$
\tilde{n}(\tilde{r})=\frac{\pi^{2}}{6 t^{2}} \sum_{k=1}^{k_{\max }} k \pi_{k} \exp \left[-\frac{k \tilde{r}^{2}}{2}\right] .
$$

where $\pi_{k}$ is determined by the formal expansion of the logarithm in Eq. (15), but also appears as a cycle weight in the path-integral representation of the bosonic density matrix where they can be measured (see the inset of Fig. 4). The successive approximations have no free parameters.

Figure 5 summarizes the density profiles of a strictly two-dimensional Bose gas in the limit $\tilde{g} \rightarrow 0$ where the classical-field calculations determine the correlation density. At the critical temperature $T_{\mathrm{KT}}$, the density in the center of the trap is critical, $\tilde{n}(0)=\tilde{n}_{c}$. Correlation effects are important only in the fluctuation regime, $\tilde{r} \lesssim 1.25 \sqrt{\tilde{g}}$, where $\tilde{n}(\tilde{r}) \gtrsim \tilde{n}_{f}$. However, the distribution of the correlation density introduces no further qualitative features to the mean-field component. The density profile may be integrated using the interpolation formula for the correlation density, Eq. (30). The critical temperature of the strictly two-dimensional Bose gas as a 


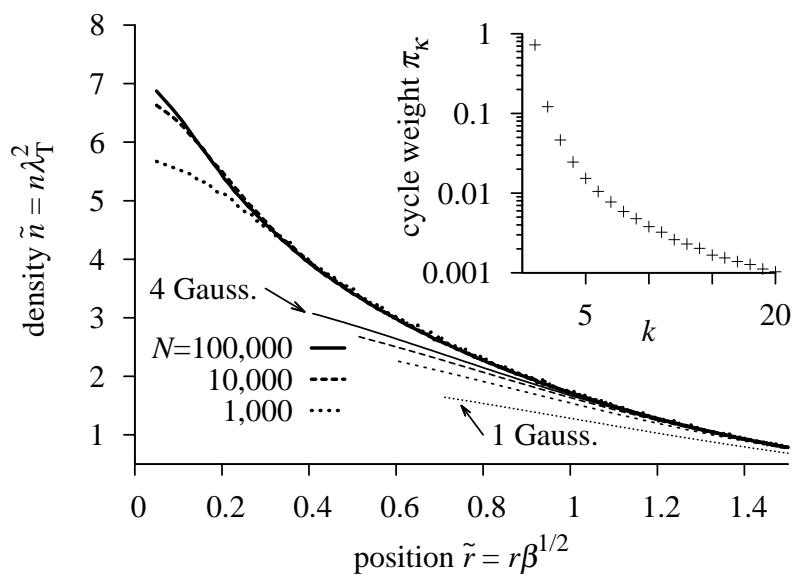

FIG. 4: QMC density profile for the NIST parameters $t=$ $T / T_{\mathrm{BEC}}^{2 \mathrm{~d}}=0.75, \tilde{g}=0.02$, and $\tilde{\omega}_{z}=0.5$, for different $N$. The data are compared to the expansion of Eq. (32), considering the largest $(k=1)$ and the four largest terms $(k=1, \ldots, 4)$. The inset shows the QMC cycle weights $\pi_{k}$ for $N=100000$ (see $[6])$.

function of the total number of particles is given by

$$
\frac{T_{\mathrm{KT}}}{T_{\mathrm{BEC}}^{2 \mathrm{~d}}} \simeq\left(1+\frac{3 \tilde{g}}{\pi^{3}} \log ^{2} \frac{\tilde{g}}{16}+\frac{6 \tilde{g}}{16 \pi^{2}}\left(15+\log \frac{\tilde{g}}{16}\right)\right]^{-1 / 2} .
$$

This expression includes correction of order $\tilde{g} \log \tilde{g}$ compared to the mean-field estimate of Refs $[6,8]$. Since corrections beyond classical-field theory are rather small (see Fig. 3), the Kosterlitz-Thouless temperature of the strictly two-dimensional trapped Bose gas is accurately described by this equation even for large coupling constants.

For general quasi-two-dimensional gases, Fig. 5 remains qualitatively correct, but the LDA mean-field density profile in the quasi-two-dimensional geometry must be used. Numerical integration of this $\tilde{g} \rightarrow 0$ density profile for the ENS parameters with $\tilde{\omega}_{z}=0.55$ and $\tilde{g}=0.13$ leads $T_{\mathrm{KT}} \simeq 0.71 T_{\mathrm{BEC}}^{2 \mathrm{~d}}$, in close agreement with $T_{\mathrm{KT}} \simeq 0.70 T_{\mathrm{BEC}}^{2 \mathrm{~d}}$ determined in Ref. [5] directly from QMC calculations using finite-size extrapolations. The quasi-two-dimensional transition temperature is smaller than the one of the strictly two-dimensional gas $\left(T_{\mathrm{KT}}^{2 \mathrm{~d}} \simeq 0.86 T_{\mathrm{BEC}}^{2 \mathrm{~d}}\right.$ for $\left.\tilde{g}=0.13\right)$. For the NIST parameters $\left(\tilde{g}=0.02, \tilde{\omega}_{z}=0.5\right)$, we have $T_{\mathrm{KT}} \simeq 0.74 T_{\mathrm{BEC}}^{2 \mathrm{~d}}$ from the integration of the $\tilde{g} \rightarrow 0$ density, and QMC data indicate a transition slightly below this value.

\section{FINITE-SIZE EFFECTS AND BOSE-EINSTEIN CROSS-OVER}

\section{A. Central coherence}

In the normal phase, the off-diagonal elements of the single-particle density matrix remain short-ranged,

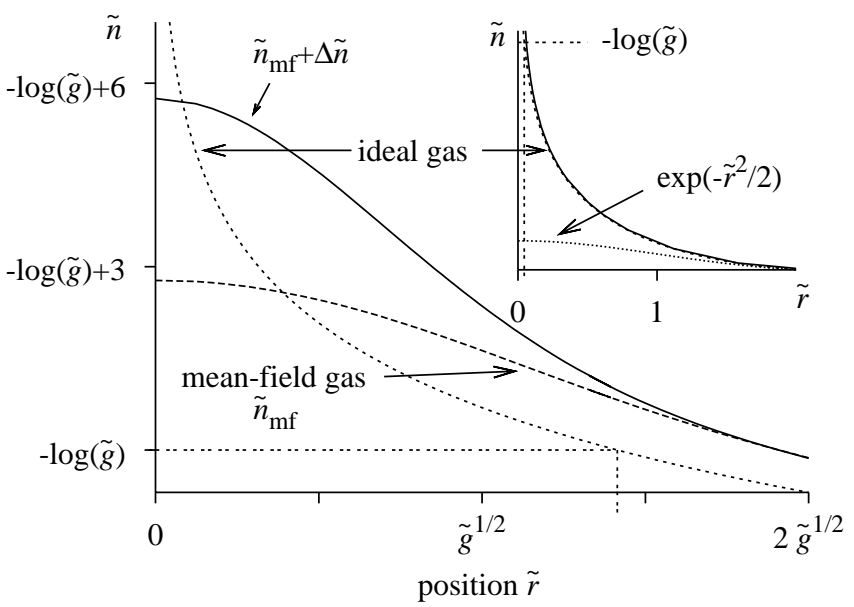

FIG. 5: Schematic density profile of a strictly two-dimensional trapped Bose gas at $T_{\mathrm{KT}}$ for $\tilde{g} \rightarrow 0$. For $\tilde{r} \gg \sqrt{\tilde{g}}, \tilde{n}$ coincides with the ideal gas at $T_{\mathrm{BEC}}^{2 \mathrm{~d}}$ (inset, the classical Boltzmann distribution $e^{-\tilde{r} 2 / 2}$ is given for comparison). In the fluctuation regime, for $\tilde{r} \lesssim 1.25 \sqrt{\tilde{g}}$, mean-field and correlation effects become important. The density diverges as $\sim \log (1 / \tilde{g})$, yet the correlation contribution $\Delta \tilde{n}$ remains finite.

so that they can be described locally. From the selfconsistent eigenfunctions of the mean-field Schrödinger equation, Eq. (12) and Eq. (13), we also obtain the offdiagonal reduced single-body density matrix:

$$
\tilde{n}_{\mathrm{mf}}^{(1)}\left(\vec{r} ; \vec{r}^{\prime}\right)=\lambda^{2} l_{z} \sum_{j} \frac{\Psi_{j}^{*}(\vec{r}) \tilde{\Psi}_{j}\left(\vec{r}^{\prime}\right)}{\exp \left(\tilde{\mu}-\beta \epsilon_{j}\right)-1} .
$$

In the local-density approximation, we can separate the contributions of the different transverse modes, and we obtain

$$
\tilde{n}_{\mathrm{mf}}^{(1)}\left(\vec{r} ; \vec{r}^{\prime}\right)=\sum_{\nu} \tilde{n}_{\mathrm{mf}, \nu}^{(1)}\left(\mathbf{r} ; \mathbf{r}^{\prime}\right) \tilde{\phi}_{\nu}(\tilde{z}) \tilde{\phi}_{\nu}\left(\tilde{z}^{\prime}\right)
$$

with

$$
\tilde{n}_{\mathrm{mf}, \nu}^{(1)}\left(\mathbf{r} ; \mathbf{r}^{\prime}\right)=\int \frac{\mathrm{d}^{2} \mathbf{k}}{(2 \pi)^{2}} \frac{\lambda_{\mathrm{T}}^{2} \mathrm{e}^{i \mathbf{k} \cdot\left(\mathbf{r}-\mathbf{r}^{\prime}\right)}}{\mathrm{e}^{\beta \hbar^{2} k^{2} / 2 m+\Delta_{\mathrm{mf}}(\tilde{r})}-1} .
$$

Here we have used that within the LDA, the density remains constant on the scale $\lambda_{\mathrm{T}}$, so that the mean-field gaps at $\tilde{r}$ and $\tilde{r}^{\prime}$ are the same.

At low densities, where the mean-field gap is large, $\Delta_{\mathrm{mf}} \gg 1$, we can expand the Bose function in Eq. (35) in powers of $\exp \left(-\Delta_{\mathrm{mf}}\right)$, and off-diagonal matrix elements rapidly vanish for distances larger than the thermal wavelength $\lambda_{\mathrm{T}}$. At higher densities, in the quantumdegenerate regime, $\Delta_{\mathrm{mf}} \ll 1$, many Gaussians contribute, and coherence is maintained over larger distances. In the limit $\Delta_{\mathrm{mf}} \rightarrow 0$, we can expand the denominator in Eq. (35), $\exp \left[\beta \hbar^{2} k^{2} / 2 m+\Delta_{\mathrm{mf}}\right]-1 \approx$ $\beta \hbar^{2} k^{2} / 2 m+\Delta_{\mathrm{mf}}$, and the off-diagonal density matrix decays exponentially. In this regime, the local mean-field coherence length is given by $\xi_{\mathrm{mf}}=\lambda_{\mathrm{T}} / \sqrt{4 \pi \Delta_{\mathrm{mf}}}$. 
In Fig. 6 and Fig. 7 we compare the normalized offdiagonal coherence function in the center of the trap

$$
c(r)=\frac{\int \mathrm{d} z n_{3 d}^{(1)}(r, z ; 0,0)}{\int \mathrm{d} z n_{3 d}^{(1)}(0, z ; 0,0)}
$$

from QMC calculations with LDA for the ENS and NIST conditions. We see that for $\tilde{n} \lesssim \tilde{n}_{f}$, as in the case of the density profile, mean-field theory accurately describes the single-particle coherence. However, it is evident that at higher densities, $\tilde{n} \gtrsim \tilde{n}_{f}$, where correlation effects for the diagonal elements of the density matrix are important, mean-field theory also fails to describe the off-diagonal matrix elements.

To characterize the decay of the off-diagonal density matrix in the fluctuation regime, $\tilde{n} \gtrsim \tilde{n}_{f}$, we consider a simple one-parameter model which neglects the momentum dependence of the self-energies in the ground state of the confining potential. The single parameter of the model, the effective local gap $\Delta(\tilde{r})$, is chosen such that it reproduces the local density of the QMC data. The density matrix of this "gap"-model, $\tilde{n}_{\Delta}^{(1)}\left(\vec{r} ; \vec{r}^{\prime}\right)=$ $\sum_{\nu} \tilde{n}_{\Delta, \nu}^{(1)}\left(\mathbf{r} ; \mathbf{r}^{\prime}\right) \tilde{\phi}_{\nu}(\tilde{z}) \tilde{\phi}_{\nu}\left(\tilde{z}^{\prime}\right)$, is a straightforward generalization of mean-field theory, where in Eq. (35), we replace

$$
\Delta_{\nu}=\left\{\begin{array}{ll}
\Delta & \text { for } \nu=0 \\
\Delta_{\mathrm{mf}} & \text { otherwise }
\end{array} .\right.
$$

To fix the gap $\Delta$ of this model, we require that the diagonal elements of the density matrix reproduces the exact density

$$
\tilde{n}_{\Delta, 0}^{(1)}(\mathbf{r} ; \mathbf{r})=\tilde{n}_{0}^{\operatorname{mf}}(\tilde{r})+\Delta \tilde{n}(\tilde{r}) .
$$

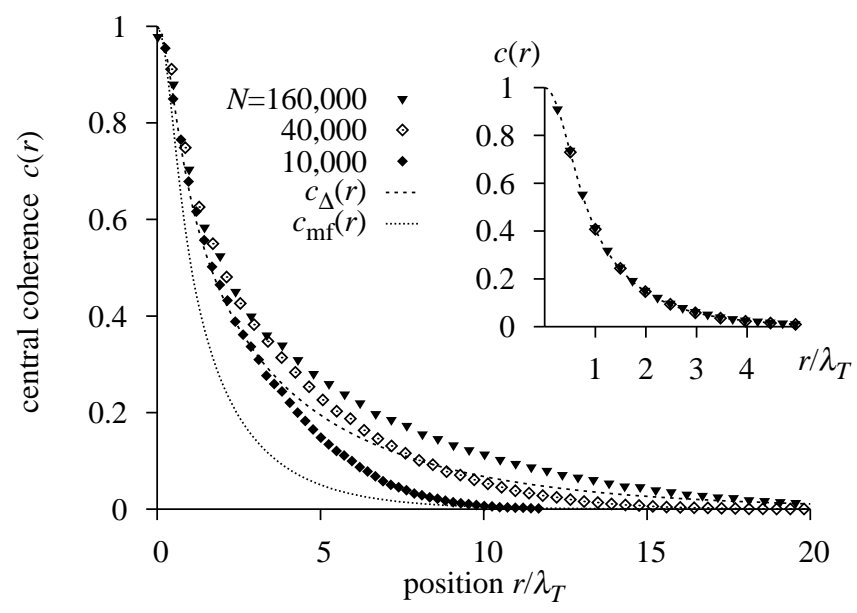

FIG. 6: Off-diagonal coherence $c(r)$ for ENS parameters with $t=0.71$ (main graph, $\tilde{n}>\tilde{n}_{f}$ ) and $t=0.769$ (inset, $\tilde{n}<\tilde{n}_{f}$ ) compared to the mean-field prediction $c_{\mathrm{mf}}(r)$ and the gap model of Eq. (37). In the fluctuation regime, finite-size effects for off-diagonal correlations are more pronounced than for the density (see Fig. 1).
Outside the fluctuation regime the gap model reduces to the mean-field limit. Inside the fluctuation regime, where a direct comparison of the coherence with meanfield theory is not very useful, the gap model provides the basis to quantify off-diagonal correlations. It cannot describe the build-up of quasi-long-range order at the Kosterlitz-Thouless transition, but its correlation length $\xi_{\Delta}=\lambda_{T} / \sqrt{4 \pi \Delta(\tilde{r})}>\xi_{\mathrm{mf}}$ bounds from below the true correlation length in the normal phase. In Fig. 6, we show that the gap model accounts for the increase of the coherence length inside the fluctuation regime, $\tilde{n}>$ $\tilde{n}_{f}$ for the ENS parameters. For smaller interactions, as in the NIST experiment, finite-size effects qualitatively change the off-diagonal elements of the density matrix (see Fig. 7).

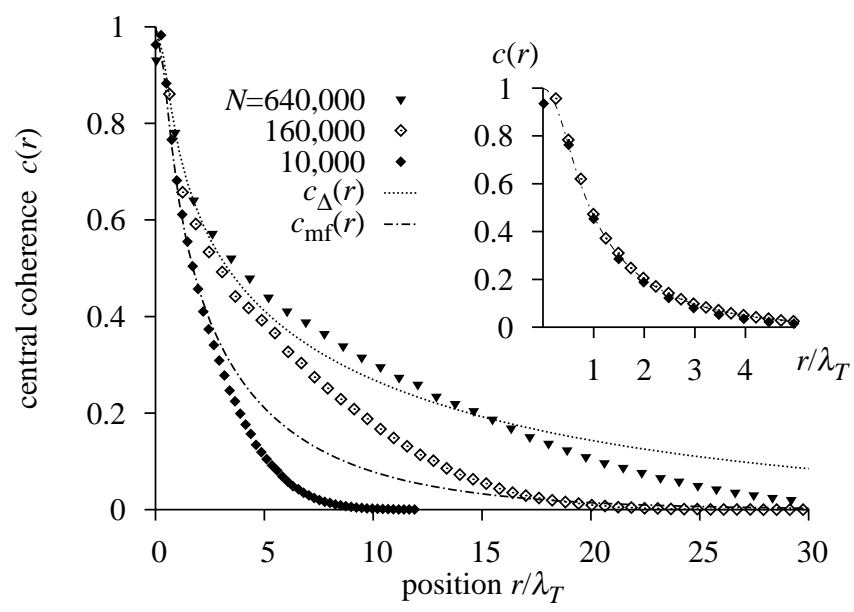

FIG. 7: Off-diagonal coherence $c(r)$ for NIST parameters with $t=0.74$ (main graph) and $t=0.769$ (inset) in comparison with the mean-field prediction, $c_{\mathrm{mf}}(r)$, and the gap model, $c_{\Delta}(r)$, defined in Eq. (37). At $t=0.769$, the total central density is $\tilde{n}(0) \simeq 5.1<\tilde{n}_{f}$, and the system is outside the fluctuation regime. At $t=0.74, \tilde{n}(0) \simeq 10.5>\tilde{n}_{f}$, and the system is close to the Kosterlitz-Thouless transition, $\Delta_{\mathrm{mf}} / \tilde{g} \simeq$ 0.08. Strong finite-size effects are evident in the fluctuation regime.

\section{B. Density profile}

Finite-size effects in the density profile are less dramatic than for the coherence (see Fig. 1). Within meanfield theory, we have compared the density profiles of the finite system directly with those in the thermodynamic limit (LDA), using the finite $N$ solution obtained by the adapted QMC calculation described in Section II B. The mean-field analysis indicates that correlation effects are at the origin of the size-effects of the full QMC density profiles in Fig. 1, in particular, at small system size, $N=1000$. 


\section{Bose-Einstein cross-over}

The finite-size effects in the coherence reflect the underlying discrete mode structure of level spacing $\sim \hbar \omega$. Off-diagonal properties for $\xi_{\Delta} \gtrsim l_{r}$ are cut off by the extension of the unperturbed ground-state wavefunction, $l_{r}=(m \omega / \hbar)^{-1 / 2}$, and resemble those of a Bosecondensed system with a significant ground-state occupation. Whereas in the thermodynamic limit, the interacting quasi-two-dimensional trapped Bose gas undergoes a Kosterlitz-Thouless phase transition, the crossover to Bose-Einstein condensation sets in when $\Delta \approx$ $\beta \hbar \omega$. If this happens outside the fluctuation regime, $\Delta_{\mathrm{mf}}^{f} \lesssim \Delta_{\mathrm{mf}} \lesssim \beta \hbar \omega$, the Bose condensation will essentially have mean-field character. Since the temperature scale is given by $\hbar \omega / T_{\mathrm{BEC}}^{2 \mathrm{~d}}=\pi / \sqrt{6 N}$, the discrete level spacing is important for small system sizes, $N \lesssim N_{\mathrm{fs}}$, with

$$
N_{\mathrm{fs}}\left(\Delta_{\mathrm{mf}}\right) \approx \frac{1}{6} \frac{\pi^{2}}{\Delta_{\mathrm{mf}}^{2} t^{2}}=\frac{\pi^{2}}{6 \tilde{g}^{2} t^{2}\left(\Delta_{\mathrm{mf}} / \tilde{g}\right)^{2}} .
$$

For small $\tilde{g}$, close to $T_{\mathrm{BEC}}^{2 \mathrm{~d}}$ where $\Delta_{\mathrm{mf}}$ is of order $\tilde{g}$, these finite-size effects trigger Bose-Einstein condensation for small $N$. In particular, for systems with $N \lesssim N_{\mathrm{fs}}\left(\Delta_{\mathrm{mf}}^{f}\right) \approx$ $\pi^{4} /\left(6 \tilde{g}^{2}\right)$, a cross-over to a mean-field-like Bose condensation occurs[31], whereas for $N \gtrsim N_{\mathrm{fs}}\left(\Delta_{\mathrm{mf}}^{c}\right) \approx 400 \tilde{g}^{-2}$ Kosterlitz-Thouless-like behavior sets in (see inset of Fig. 10). We notice that the finite-size scale $N_{\mathrm{fs}} \propto 1 / g^{2}$ diverges very rapidly with vanishing interactions, which could make the cross-over experimentally observable.

For a finite system with $N \lesssim N_{\mathrm{fs}}\left(\Delta_{\mathrm{mf}}^{f}\right)$, the condensate wavefunction does not develop immediately a ThomasFermi shape, but remains close to the Gaussian groundstate wavefunction of the ideal gas with typical extension $l_{r}=(m \omega / \hbar)^{-1 / 2}$. Thus, for small condensate fraction $n_{0}$, deviations of the moment of inertia $I$ of the trapped gas from its classical value, $I_{\mathrm{cl}}=\int \mathrm{d}^{2} \mathbf{r} r^{2} n(r) \sim N l_{T}^{2}$, are negligible, of order $\left(I_{\mathrm{cl}}-I\right) / I_{\mathrm{cl}} \sim n_{0} l_{r}^{2} / l_{T}^{2} \sim N^{-1 / 2}$. Only for larger condensates with $\tilde{g} N_{0} \gg 2 \pi$, the selfinteraction energy dominates the kinetic energy, and the condensate wavefunction approaches the Thomas-Fermi distribution of radius $\sim l_{T}$, resulting in a non-classical value of the moment of inertia. In this low-temperature regime, the system can be described by a condensate with a temperature-dependent fluctuating phase [13]. Therefore, for small systems, a non-classical moment of inertia only occurs at lower temperatures than condensation, roughly, at a condensate fraction $n_{0} \gtrsim \tilde{g}$.

To illustrate the cross-over between the Bose-Einstein regime at small $N$ and the Kosterlitz-Thouless regime at large $N$, we have calculated the condensate fraction and condensate wavefunction for the ENS parameter in Fig. 8. To determine both quantities in inhomogeneous systems, $n_{3 d}^{(1)}\left(\vec{r}, \vec{r}^{\prime}\right)$ must be explicitly diagonalized, as the eigenfunctions of the single-particle density matrix are not fixed by symmetry alone. In quasi-two-dimensional systems, the full resolution of the off-diagonal density ma- trix in the tightly confined $z$-direction is difficult. It is more appropriate to consider the in-plane density matrix, $n^{(1)}\left(\mathbf{r}, \mathbf{r}^{\prime}\right)$, where the confined direction is integrated over. $n^{(1)}\left(\mathbf{r}, \mathbf{r}^{\prime}\right)=\int \mathrm{d} z \int \mathrm{d} z^{\prime} n_{3 d}^{(1)}\left(\mathbf{r}, z ; \mathbf{r}^{\prime}, z^{\prime}\right)$. Because of rotational symmetry, $n^{(1)}\left(\mathbf{r}, \mathbf{r}^{\prime}\right)$ is block-diagonal in angularmomentum Fourier components

$$
n^{(1)}\left(\mathbf{r}, \mathbf{r}^{\prime}\right)=\sum_{n=0}^{\infty} \sum_{l=-\infty}^{\infty} N_{n l} \varphi_{n l}^{*}\left(r^{\prime}\right) \varphi_{n l}(r) e^{l \alpha\left(\mathbf{r}, \mathbf{r}^{\prime}\right)}
$$

where $\alpha\left(\mathbf{r}, \mathbf{r}^{\prime}\right)$ denotes the angle between $\mathbf{r}$ and $\mathbf{r}^{\prime}$, and $N_{n l}$ is the occupation number of the normalized eigenmode $\varphi_{n l}$. The (in-plane) condensate fraction, $n_{0}=$ $N_{00} / N$, corresponds to the largest eigenvalue with $l=0$ and condensate wavefunction $\varphi_{0}(r)=\varphi_{00}(r)$. Projection on the Fourier components is convenient for determining the condensate fraction and wavefunction within QMC.

For the ENS parameters in Fig. 8, the central density is already inside the fluctuation regime, and the condensate wavefunction differs from the Gaussian ground state of an ideal gas. However, for small systems, $N \lesssim 10^{3}$, it still has a Gaussian shape indicating that the condensate kinetic energy dominates the potential energy. The condensate fraction vanishes as $n_{0} \sim N^{-1 / 2}$.

The QMC calculations of [5] demonstrated that the condensate fraction of the quasi-two-dimensional Bose gas vanishes in the normal and in the superfluid phase in the thermodynamic limit, $N \rightarrow \infty$. However, in the low-temperature superfluid phase, the condensate fraction approaches zero very slowly with increasing system size, so that an extensive condensate remains for practically all mesoscopic systems.

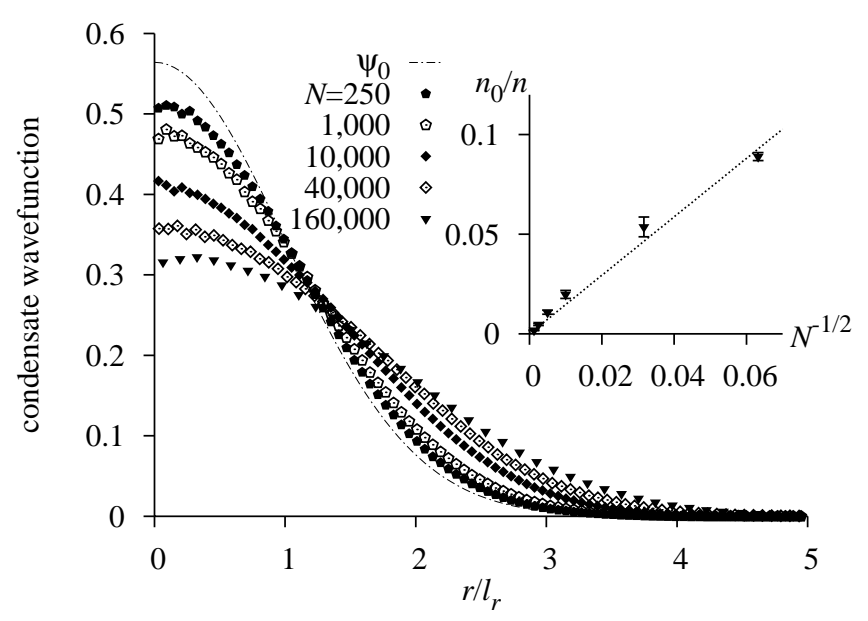

FIG. 8: Condensate fraction $n_{0}$ (inset) and wavefunction $\varphi_{0}(r)$ (main graph), computed by QMC for ENS parameters with $t=0.71$ for various system sizes. The condensate wavefunction is modified with respect to the ground-state wavefunction $\psi_{0}(r)$ of the unperturbed harmonic oscillator, but the Gaussian shape is preserved. The two largest systems are above the scale $N_{\text {fs }}$ (see Eq. (39)) 


\section{TWO-PARTICLE CORRELATIONS}

\section{A. Pair-correlation function}

Density-density correlations can be analyzed by considering the three-dimensional pair-correlation function, $n^{(2)}\left(\vec{r} ; \vec{r}^{\prime}\right)$. This quantity factorizes within mean-field theory into terms described by the one-particle density matrix, $n_{\mathrm{mf}}^{(1)}\left(\vec{r}_{1} ; \vec{r}_{2}\right)$ (see Section IV A):

$$
n_{\mathrm{mf}}^{(2)}\left(\vec{r}_{1} ; \vec{r}_{2}\right)=n_{\mathrm{mf}}\left(\vec{r}_{1}\right) n_{\mathrm{mf}}\left(\vec{r}_{2}\right)+\left[n_{\mathrm{mf}}^{(1)}\left(\vec{r}_{1} ; \vec{r}_{2}\right)\right]^{2} .
$$

For vanishing distances, $\vec{r}_{1} \rightarrow \vec{r}_{2}$, mean-field theory pre$\operatorname{dicts} n_{\mathrm{mf}}^{(2)}(\vec{r}, \vec{r})=2 n_{\mathrm{mf}}^{2}(\vec{r})$. For Bose-condensed atoms, this bunching effect is absent.

In two dimensions, deviations from $2 n^{2}$ of the paircorrelation function at contact signal beyond-mean-field fluctuations $[12,19,21]$. In Ref. [12], the universal character of the contact value was used to define the quasicondensate density, $n_{\mathrm{qc}}(r) \equiv\left[2[n(r)]^{2}-n^{(2)}(r, r)\right]^{1 / 2}$. This quantity has been studied in Ref. [22] for a quasitwo-dimensional trapped gas within classical-field theory.

The pair-correlation function of the quasi-twodimensional gas is obtained by integrating both coordinates over the confined direction

$$
n^{(2)}\left(\mathbf{r}_{1} ; \mathbf{r}_{2}\right)=\int \mathrm{d} z_{1} \int \mathrm{d} z_{2} n^{(2)}\left(\mathbf{r}_{1}, z_{1} ; \mathbf{r}_{2}, z_{2}\right),
$$

and mean-field expressions for this quantity follow from Eq. (41) together with Eqs (34) and (35). Inside the fluctuation regime, the gap model (using Eq. (37) in the mean-field expressions) again leads to an improved paircorrelation function $n_{\Delta}^{(2)}$.

Figure 9 illustrates that outside the fluctuation regime mean-field theory describes the pair-correlation function well. In contrast to a strictly two-dimensional gas, the contact value of the pair correlation function is below 2 . Even in the mean-field regime, the occupation of more than one mode in the confined direction causes a noticeable reduction of the pair-correlation function at contact. The above definition of the quasi-condensate must therefore be modified in this geometry to maintain its universal character.

At short distances $r \sim r_{0}$, the pair-correlation function depends on the specific form of the interaction. This cannot be reproduced by the single-particle mean-field approximation. However, two-particle scattering properties dominate for small enough distances as, for example, the wavefunction of hard spheres must vanish for overlapping particles. This feature can be included in mean-field theory by multiplying its pair-correlation functions by a short-range term $\chi_{2 d}(r)$, which accounts for two-particle scattering[23]. In two dimensions, $\chi_{2 d}$ shows a characteristic logarithmic behavior for short distances:

$$
\chi_{2 d}(r \rightarrow 0) \simeq\left[1+\frac{\tilde{g}}{2 \pi} \log \sqrt{\frac{\pi e^{C}}{2}} \frac{r}{\lambda_{\mathrm{T}}}\right]^{2}
$$

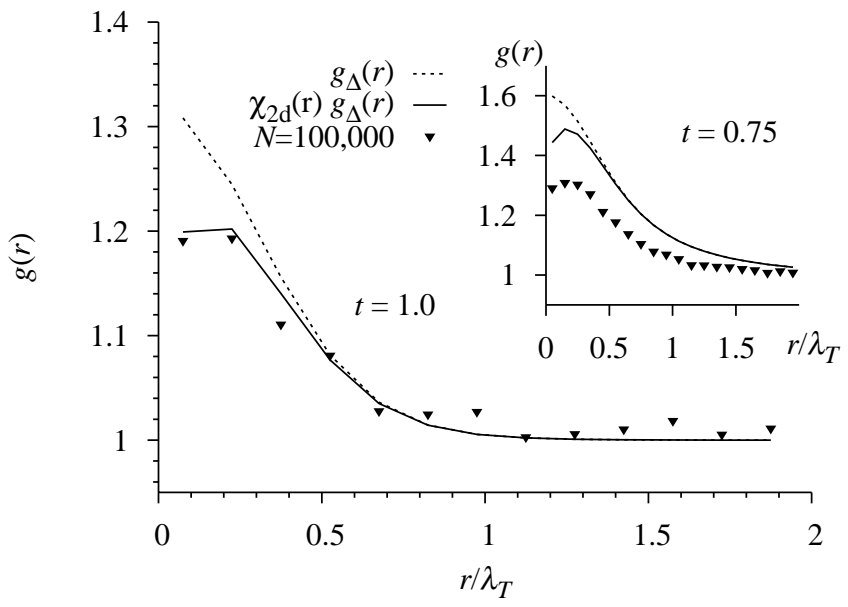

FIG. 9: Central pair correlations, $g(r)=n^{(2)}(r, 0) /[n(r) n(0)]$, of the quasi-two-dimensional trapped Bose gas at temperature $T=T_{\mathrm{BEC}}^{2 \mathrm{~d}}$ (main figure) and $T=0.75 T_{\mathrm{BEC}}^{2 \mathrm{~d}}$ (inset) for $N=$ 100, 000 atoms (ENS parameters), together with the prediction of the mean-field gap model, $g_{\Delta}(r)=n_{\Delta}^{(2)}(r, 0) / n(r) n(0)$, and the short-range improved mean-field model, $\chi_{2 d}(r) g_{\Delta}(r)$.

Factorizing out the short-range behavior from the paircorrelation function, the correlation part of the renormalized pair-correlation function, $\tilde{n}_{\Delta}^{(2)}-\tilde{n}^{(2)} / \chi_{2 d}$, should be dominated by contributions from classical-field theory, its contact value is universal, and it might be used to define a quasi-condensate density in quasi-two dimensions via $\tilde{n}_{\mathrm{qc}}^{2}=\lim _{r \rightarrow 0}\left[\tilde{n}_{\Delta}^{(2)}(r, 0)-\tilde{n}^{(2)}(r, 0) / \chi_{2 d}(r)\right]$ (see Fig. 10). Similar to the correlation density, the quasi-condensate density is universal. At $T_{\mathrm{KT}}$, the classical field result is $\tilde{n}_{\mathrm{qc}} \simeq 7.2$, whereas it is around 2.7 at the onset of the fluctuation regime, so that, in the normal phase, $n_{\mathrm{qc}} / n$ vanishes as $|\log \tilde{g}|^{-1}$ for $\tilde{g} \rightarrow 0$.

\section{B. Local-density correlator}

Due to the three-dimensional nature of the underlying interaction potential, observables which couple directly to local three-dimensional density fluctuations involve the following density correlator

$$
K^{(2)}=\lim _{\delta \rightarrow 0} \frac{\sqrt{2 \pi} l_{z} \int \mathrm{d} z n^{(2)}(\mathbf{r}, z ; \mathbf{r}+\delta, z)}{\chi_{3 d}(\delta)},
$$

where $\chi_{3 d}\left(r \ll \lambda_{\mathrm{T}}\right) \simeq\left(1-a_{s} / r\right)^{2}$ describes the universal short-distance behavior of the three-dimensional two-body wavefunction in terms of the $s$-wave scattering length $a_{s}$.

Arguments similar to those in Section VA show that the local-density correlator in general differs from the contact value of the quasi-two-dimensional paircorrelation function, Further, the integration over the square of the ground state density in $z$ leads to a 


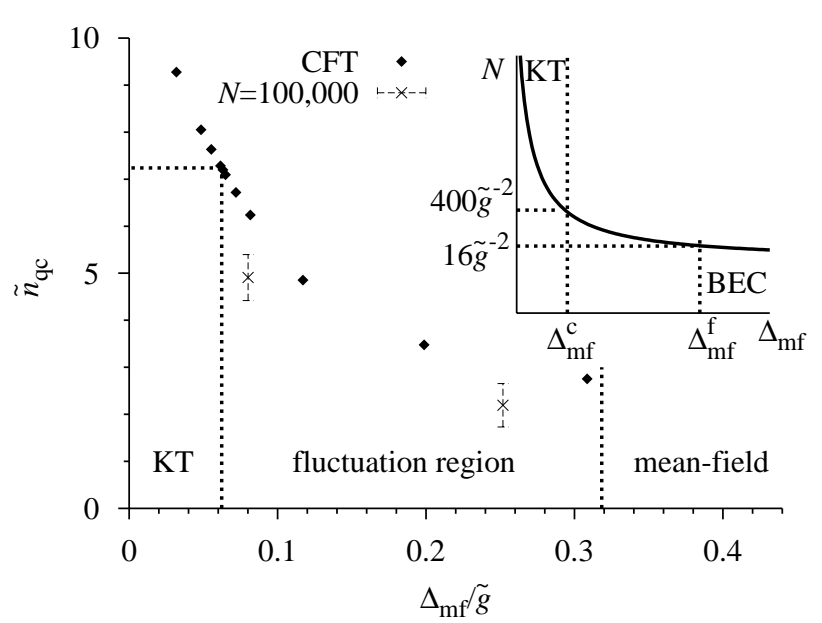

FIG. 10: Quasi-condensate density $n_{\mathrm{qc}}$ obtained by QMC from the renormalized pair-correlation function at $T=$ $0.71 T_{\mathrm{BEC}}^{2 \mathrm{~d}}$, and $T=0.75 T_{\mathrm{BEC}}^{2 \mathrm{~d}}$ (see Fig. 9) for ENS parameters, plotted as a function of $\Delta_{\mathrm{mf}}$, and compared to classicalfield simulations [12]. The inset shows the boundary of the region with strong finite-size effects (see Eq. (39)).

phase-space-density dependence which destroys the simple mean-field property $K^{(2)} \propto 2 n$ of strictly twodimensional Bose gases.

\section{CONCLUSIONS}

In this paper, we have studied the quasi-twodimensional trapped Bose gas in the normal phase above the Kosterlitz-Thouless temperature for small interactions $\tilde{g}<1$. We have discussed the three qualitatively distinct regimes of this gas: For phase-space densities $\tilde{n} \lesssim 1$, it is classical. At higher density, $1 \lesssim \tilde{n} \lesssim \tilde{n}_{f}$, the gas is in the quantum mean-field regime, and its coherence can be maintained over distances much larger than $\lambda_{\mathrm{T}}$. Finally, mean-field theory fails in the fluctuation regime $\tilde{n}_{f} \lesssim \tilde{n} \leq \tilde{n}_{c}$ and beyond-mean-field corrections must be taken into account.

In the fluctuation regime, for small interactions, the deviations of the density profile with respect to the mean- field profile are universal. Mean-field theory thus accounts for most microscopic details of the gas (which depend on the interactions and on the trap geometry). We have shown in detail how to extract the correlation density (the difference between the density and the meanfield density at equal chemical potential) from QMC density profiles and the LDA mean-field results, and compared it to the universal classical field results. Quantum corrections to the equation of state, expected of order $\tilde{g}$, were demonstrated to be small for current experiments with $\tilde{g} \lesssim 0.2$. The smooth behavior of quantum corrections, which has been already noticed in QMC calculations of the Kosterlitz-Thouless transition temperature in homogeneous systems [24], strongly differs from the three-dimensional case $[25,26]$ where quantum corrections to universality are non-analytic [27, 28], and where the universal description holds only asymptotically. It would be interesting if these universal deviations from mean-field theory could be observed experimentally.

Correlation effects in local observables, e.g. the density profile, and local-density correlators, converge rather quickly to their thermodynamic limit value, and correlation effects for mesoscopic systems are well described by the local-density approximation. Off-diagonal coherence properties show much larger finite-size effects, in particular for weak interactions. This introduces qualitative changes for mesoscopic system sizes and, in particular, the cross-over between Bose-Einstein physics at small particle number $N \lesssim$ const $/ \tilde{g}^{2}$ and the KosterlitzThouless physics for larger systems. Tuning the interaction strength via a Feshbach resonance might make it possible to observe the cross-over between Bose-Einstein condensation and Kosterlitz-Thouless physics in current experiments.

\section{Acknowledgments}

W. K. acknowledges the hospitality of Aspen Center for Physics, where part of this work was performed.

NB: The computer programs used in this work are available from the authors.
[1] Z. Hadzibabic, P. Krüger, M. Cheneau, B. Battelier, and J. Dalibard, Nature 441, 1118 (2006).

[2] P. Clade, C. Ryu, A. Ramanathan, K. Helmerson, W.D. Phillips Phys. Rev. Lett. 102, 170401 (2009).

[3] J. M. Kosterlitz and D. J. Thouless, J. Phys. C 6, 1181 (1973); J. M. Kosterlitz, J. Phys. C 7, 1046 (1974); V. L. Berezinskii, Sov. Phys. JETP 32, 493 (1971); 34, 610 (1972).

[4] P. Krüger, Z. Hadzibabic, and J. Dalibard, Phys. Rev. Lett. 99, 040402 (2007).

[5] M. Holzmann and W. Krauth, Phys. Rev. Lett. 100
190402 (2008)

[6] M. Holzmann, M. Chevallier, and W. Krauth, EPL 82 30001 (2008).

[7] D.S. Fisher and P.C. Hohenberg, Phys. Rev. B 37, 4936 (1988).

[8] M. Holzmann, G. Baym, J.-P. Blaizot, and F. Laloë, Proc. Nat. Acad. Sci. 104, 1476 (2007).

[9] N. Prokof'ev, O. Ruebenacker, and B. Svistunov, Phys. Rev. Lett. 87, 270402 (2001).

[10] Z. Hadzibabic, P. Krüger, M. Cheneau, S. P. Rath, and J. Dalibard, New J. Phys 10045006 (2008). 
[11] R. N. Bisset, D. Baillie, and P. B. Blakie, Phys. Rev. A 79, 013602 (2009).

[12] N. Prokof'ev and B. Svistunov, Phys. Rev. A 66, 043608 (2002).

[13] D. S. Petrov, M. Holzmann, and G. V. Shlyapnikov, Phys. Rev. Lett. 84, 2551 (2000).

[14] D. S. Petrov and G. V. Shlyapnikov, Phys. Rev. A 64, 012706 (2001).

[15] L.-K. Lim, C. M. Smith, and H.T.C. Stoof, Phys. Rev. A 78, 013634 (2008).

[16] W. Krauth, Phys. Rev. Lett. 77, 3695 (1996).

[17] M. Holzmann, W. Krauth, and M. Naraschewski, Phys. Rev. A 59, 2956 (1999).

[18] W. Krauth, Statistical Mechanics: Algorithms and Computations, Oxford University Press (Oxford, UK) (2006).

[19] Yu. Kagan, V.A. Kashurnikov, A.V. Krasavin, N.V. Prokof'ev, and B.V. Svistunov, Phys. Rev. A 61, 43608 (2000).

[20] M. Chevallier and W. Krauth, Phys. Rev. E 76051109 (2007).

[21] L. Giorgetti, I. Carusotto, and Y. Castin, Phys. Rev. A 76, 013613 (2007).

[22] R.N. Bisset, M.J. Davis, T.P. Simula, and P.B. Blakie, Phys. Rev. A 79, 033626 (2009).

[23] M. Holzmann and Y. Castin, Eur. Phys. J. D 7, 425 (1999).
[24] S. Pilati, S. Giorgini, and N. Prokof'ev, Phys. Rev. Lett. 100, 140405 (2008).

[25] G. Baym, J.-P. Blaizot, M. Holzmann, F. Laloë, and D. Vautherin, Phys. Rev. Lett. 83, 1703 (1999); Eur. Phys. J. B24, 107 (2001).

[26] M. Holzmann and G. Baym, Phys. Rev. Lett. 90, 040402 (2003).

[27] M. Holzmann, G. Baym, J.-P. Blaizot, and F. Laloë, Phys. Rev. Lett. 87, 120403 (2001).

[28] P. Arnold, G. Moore, and B. Tomásîk, Phys. Rev. A 65, 013606 (2002).

[29] In general, at equal chemical potential, the mean-field density differs from the exact one, and so does also the total number of particles in the trap.

[30] Similar to the mean-field gap, one may introduce an effective mean-field coupling constant $\tilde{g}_{\mathrm{mf}}=$ $\tilde{g} \int \mathrm{d} \tilde{z}\left|\tilde{\phi}_{0}(\tilde{z})\right|^{4} / \int \mathrm{d} \tilde{z}\left|\tilde{\phi}_{0}(\tilde{z})\right|^{2}$ which accounts for modifications of the in-plane interactions in the ground state of the confining potential. This leads to small corrections, not visible for the experimental parameters considered in this paper.

[31] In contrast to the infinite mean-field gas, these finite mean-field systems undergo a Bose-Einstein condensation slightly below $T_{\mathrm{BEC}}^{2 \mathrm{~d}}[8]$. 